\title{
Les migrations et le marché de soins aux personnes âgées
}

\author{
Guita Grin Debert \\ Unicamp, Institut de Philosophie et Sciences Sociales, Département d'Anthropologie, \\ Campinas/SP, Brazil
}

\begin{abstract}
This article focuses on the migration of women from Latin America and Eastern Europe to work with home care of the elderly in Italy. Through an ethnographic approach and based on field research conducted in Bologna with caregivers and their employers the aim of this paper is to discuss: (1) the configuration of this new growing market that responds to the rapid aging of the world's population; (2) how gender, age and nationality produce categories of differentiation and inequality; (3) how the visibility gained by the duo combining two unwanted populations - the elderly and immigrants - redefines forms of dependence, gives new meaning to family relationships, to obligations of the state and to domestic life.
\end{abstract}

Keywords: home care, elderly, immigration, family relationships, Bologna, Italy.

\section{Résumé}

Cet article aborde l'immigration de femmes d'Amérique latine et d'Europe de l'Est allant travailler dans les soins à domicile de personnes âgées en Italie. Fondé sur une recherche de terrain menée à Bologne auprès de soignants et de leurs employeurs, il discute: (1) les configurations de ce nouveau marché en expansion qui répond au vieillissement rapide de la population mondiale; (2) la façon dont genre, âge et nationalité produisent des catégories de différenciation et d’inégalité; (3) la manière dont la visibilité acquise par ces deux populations indésirables, personnes âgées et ,immigrés, redéfinit les formes de dépendance et donne de nouveaux sens aux relations familiales, aux droits et obligations de l'État et à la vie domestique.

Mots-clés: soins à domicile, personnes âgées, migration, relations familiales, Bologne, Italie.

\section{Resumo}

O artigo focaliza a imigração de mulheres da América Latina e da Europa do leste para trabalhar com o cuidado à domicílio de idosos na Itália. Tendo como base uma etnografia realizada na cidade de Bolonha com cuidadoras e seus empregadores, o objetivo do artigo é discutir: (1) as configurações deste novo mercado em expansão que responde ao envelhecimento acelerado da população mundial; (2) o modo pelo qual gênero, idade e nacionalidade produzem categorias de diferenciação e de desigualdade; (3) o modo como a visibilidade deste duo envolvendo grupos discriminados - imigrantes e idosos - redefine formas de dependência e dá novos significados às relações familiares, aos direitos e obrigações do estado e à vida doméstica.

Palavras-chave: cuidado domiciliar, idosos, migração, relações familiares, Bolonha, Itália. 


\section{Les migrations et le marché de soins aux personnes âgées}

\section{Guita Grin Debert}

Le paysage des centres urbains, en particulier dans les quartiers les plus riches, est de plus en plus marqué par la présence d'hommes et de femmes âgés accompagnés par des femmes immigrées, venues d'autres pays ou de régions plus pauvres du même pays, chargées de leurs soins. Cet article, qui prend pour référence l'insertion des femmes d'Amérique latine et d'Europe de l'Est sur le marché italien de soins aux personnes âgées et se fonde sur une recherche de terrain menée à Bologne auprès de soignants et de leurs employeurs, discute: (1) les configurations de ce nouveau marchét en expansion qui répond à l'augmentation de la durée de vie et donne de nouvelles significations aux migrations; (2) la façon dont genre, âge et nationalité produisent des catégories de différenciation et d'inégalité à partir des nouvelles relations contractuelles de travail et d'affection ainsi constituées; (3) la façon dont la visibilité acquise par ces deux populations indésirables, personnes âgées et immigrés, redéfinit les formes prises par la dépendance et donne un contenu et des sens nouveaux aux relations familiales, aux droits et obligations de l'État, à la vie domestique et à la présence de ces nouveaux acteurs dans les espaces publics.

Le rapport entre immigration et vieillissement de la population est un sujet encore peu étudié. La longévité accrue et la baisse des taux de fécondité posent la question: quelle main-d’œuvre prendra soin des personnes âgées ou, comme le disait un étudiant en gérontologie: "Qui, dans l'avenir, poussera les fauteuils roulants des personnes âgées?” Dans quelle mesure l’immigration aura-t-elle un poids dans la réponse à cette question majeure?

L’immigration fait croître la quantité de jeunes travailleurs au sein d'une population nationale de plus en plus vieillie. Les immigrés seront-ils l'avant-garde des soins pour les personnes âgées? Comment peut-on expliquer les politiques menées pour freiner l'immigration qui mobilise un contingent croissant de partisans?

Nous savons que les pays d'Amérique latine, qui, jusqu'à récemment, étaient des pays d'accueil d'immigrés, sont touchés par des vagues croissantes d'émigration de leur population vers l'Amérique du Nord et l'Europe. Les femmes d'Amérique latine font partie de cette vague d'étrangers parmi lesquels la population européenne et nord-américaine choisit ses aides-soignantes.

Pour discuter l'incidence des formes contemporaines de gestion de la dépendance sur les nouvelles configurations de l'immigration et du travail du soin, je commencerai par présenter des approches sociologiques de type structurel pour comprendre la connexion entre mondialisation et vieillissement. Je reprendrai ensuite les récits des aides-soignantes sur leurs expériences de travail en

\footnotetext{
1 Par marché de soins aux personnes âgées, on désigne un marché du travail théorique où se rencontrent l'offre et la demande de travail de soins pour les personnes âgées. L’offre de travail de soins provient de salariés et de personnes à la recherche d'emploi et la demande de travail des besoins d'entreprises ou de personnes physiques. Dans cet article, le marché abordé est composé de femmes immigrées qui, en échange d'un salaire, cherchent un travail de soins chez des personnes âgées dépendantes et sont employées par leurs enfants, leur famille ou des connaissances afin d’habiter avec elles et de les accompagner dans leurs activités de la vie quotidienne, comme par exemple se changer, faire sa toilette, śalimenter et prendre ses médicaments.
} 
Italie. Enfin, je me concentrerai sur la façon dont les constructions de genre et de nationalité interviennent dans les interactions entre employeurs et immigrées, et entre immigrées de différentes régions et nationalités. ${ }^{2}$

Nous rappelons que Bologne est une ville italienne d'environ 386 ooo habitants, située dans le nord-est du pays. Il s'agit de l'une des sept plus grandes villes d'Italie et sa population étrangère résidente était de 48466 personnes lors du dernier recensement (2010).

Les données présentées ci-dessous se basent sur une recherche qualitative comprenant des entretiens et une observation des comportements d'aides-soignantes de personnes âgées ayant émigré d'Amérique latine et d'Europe de l'Est vers l'Italie, ainsi que de leurs employeurs italiens. L'enquête sur les aides-soignantes péruviennes et équatoriennes a été menée à partir d'entretiens réalisés lors de la messe du dimanche, à l'église de San Donato à Bologne, où j'avais observé la présence de ces immigrées. Les entretiens avec des femmes d'Europe de l'Est ont commencé dans un parc public de Bologne où j'ai pu entrer en contact avec un réseau d'aides-soignantes venues de cette région, en particulier de Moldavie. La recherche concernant les employeurs a été réalisée auprès de connaissances personnelles, principalement des professeurs et fonctionnaires de 1’Université de Bologne, en Italie, où j’ai donné le cours Genre et Vieillesse, en 2010.3

\section{Vieillissement, Biopolitique et Mondialisation}

As the rich economies become richer they become more desirable and as they raise their walls to keep immigrants and refugees out, they feed the illegal trade in people. Yet even as the rich countries try harder and harder to keep would-be immigrants and refugees out, they face a growing demographic deficit and rapidly aging populations. According to a major study by the Austrian at the end of the current century, population size in Western Europe will have shrunk by 75 million (under current fertility and immigration patterns) and almost 50 percent will be over 60 years old - a first in its history. Where will they get the new young workers they need to support the growing elderly population and to do the unattractive jobs whose numbers are growing, some of which will involve home and institutional care for old people? Export of older people and of economic activities is one option being considered now. But there is a limit to how many old people and low wage jobs you can export. It looks like immigration will be part of the solution. But the way the countries in the global north are proceeding is not preparing them to handle this. They are building walls to keep would-be immigrants out, thereby feeding illegal trafficking. (Saskia Sassen, 2001 cité par Brett Neilson, 2003)

J'emprunte ce sous-titre d'un article de Brett Neilson (2003) qui, en prenant pour référence les commentaires de Foucault sur le biopouvoir et ceux d'Agamben sur l'homo sacer, discute l'imbrication de la mondialisation et du vieillissement.

Les flux de personnes, de biens, d'argent et de technologies traversent les frontières nationales avec une relative facilité et défient la souveraineté des États. Les politiques d'immigration, la biotechnologie, la déréglementation des marchés financiers et les nouveaux modes d'organisation du monde du travail ont des implications importantes sur la vieillesse et le vieillissement de la population menace les États nationaux pour ce qui est de la relation entre les travailleurs payant des impôts et les retraités.

La dynamique des marchés financiers est dépendante du capital accumulé par les caisses de retraite et,

\footnotetext{
2 La recherche ici présentée a été financée par la Fondation de soutien à la recherche de l'état de Sao Paulo (FAPESP: 2011/10537-6) ainsi que par le Conseil national de développement scientifique et technologique (CNPq 303756/2013).

3 Le cours fait partie du Programme Gemma-Erasmus Mundus Master's Degree in Women's and Gender Studies in Europe
} 
comme le montre Saskia Sassen, les jeunes chômeurs et les immigrés originaires de pays pauvres tentent de franchir les barrières posées par les pays riches, qui ont des populations à fort taux de vieillissement et ils finissent par occuper une position importante dans l'offre de travail de soins aux personnes âgées.

Par le terme "biopouvoir", Foucault cherche à souligner, dans le premier volume de l'Histoire de la Sexualité, les discontinuités de l'exercice du pouvoir. Jusqu'au XVIII siècle, l'auteur montre que le souverain avait pouvoir de vie et de mort sur ses sujets. $S$ 'il se sentait menacé par ses ennemis, il avait le droit d'appeler ses sujets à la guerre. Selon Paul Rabinow et Nikolas Rose (2006), ce modèle de souveraineté est resté pratiquement inchangé dans la philosophie politique. Pour Foucault (1976), cette puissance est néanmoins remplacée par le biopouvoir ou la biopolitique, dont la tâche est d'assurer et de gérer la vie des citoyens. Pour garantir le bien-être de la population, un ensemble de dispositifs a été créé afin de classer la population et de gérer la vie de tous et chacun. Les statistiques, le plan urbain et la gestion de la santé publique sont des expressions de cette discontinuité dans l'exercice du pouvoir souverain ; tout comme la constitution d'un segment particulier de la population et la formation de spécialistes chargés d'analyser les besoins de la vieillesse et de proposer les moyens de les résoudre sont des expressions du biopouvoir qui légitiment les États modernes.

Agamben (2002) tente de développer ces paramètres en utilisant la figure de l'homo sacer pour répondre à la manière dont la vie humaine doit être incluse dans le calcul de la puissance souveraine. L'homo sacer est une figure du droit romain archaïque désignant quelqu'un ayant été jugé et reconnu coupable d'un crime. Or, comme cette personne ne peut pas être sacrifiée, qui la tue ne commet pas un crime en soi. Par cette image, Agamben suggère que le biopouvoir dans la modernité marque la manière dont la vie biologique, la "vie nue", la vie qui peut être effacée, entre dans la politique. Les pratiques d'hygiène et les camps de concentration nazi sont paradigmatiques des nouvelles formes de domination visant à éliminer tout ce qui est étranger et peut contaminer la santé de la population. La souveraineté des États dépend de la capacité de suspendre la loi, de la création d'états d'exception, au nom de la défense de la citoyenneté et de la sécurité nationale. Les États modernes sont, par conséquent, des états d'exception. Le souverain est à la fois à l’intérieur et à l'extérieur du système juridique, une zone de différenciation qui permet d'être au sein et en dehors de la loi. La logique disciplinaire d'aujourd'hui se serait répandue au point de faire d'un citoyen un homo sacer.

Paul Rabinow et Nikolas Rose (2006) mettent en évidence la difficulté d'utiliser un seul schéma, comme celui d'Agamben, pour analyser diverses situations marquant le monde contemporain, des tueries au Rwanda à l'épidémie de sida en Afrique.

Ils considèrent à juste titre que l'essence de la pensée critique est la capacité de faire des distinctions permettant d'évaluer précisément la particularité des situations et ils suggèrent que, contrairement à une vision monolithique du pouvoir et de la domination, un empirisme modeste, attentif aux spécificités locales, aux petites différences, aux moments de changement dans la relation entre pouvoir et savoir, peut faire la différence en révélant des paramètres pas nécessairement appropriés à des modèles absolus et homogènes.

L’analyse de Neilson est séduisante parce qu'elle se confronte à la situation des immigrés et des réfugiés, laquelle, comme le montre l'auteur, a pour effet d'augmenter la répartition des richesses et du travail dans le monde mondialisé, ce qui pousse un nombre croissant des personnes à tenter de franchir les barrières posées par les pays développés.

Ce mouvement, du fait des systèmes de détention et de déportation existant, finit par créer des systèmes de travail proches des pratiques esclavagistes. Loin d'être des formes archaïques, ces modalités de travail sont constitutives du développement capitaliste qui vise à limiter la mobilité des travailleurs par le remplacement continu des personnes prêtes à accepter un travail faiblement rémunéré, comme c'est le cas du soin aux personnes âgées. Dans ce processus, de nouveaux groupes d’immigrants remodèlent le paysage des villes en 
offrant un flux régulier de jeunes vers les pays du Nord. ${ }^{4}$ Les travailleurs migrants vivent dans une situation d'incertitude complète parce qu'ils toujours vus comme des illégaux, des criminels, voire des terroristes potentiels. 5

En ce sens, ils sont une sorte d'"homo sacer" selon la définition d'Agamben.

Dans un texte intitulé "L'amour et l'or", Arlie Hochschild (2003) évoque de façon succincte les changements dans les relations entre pays développés et pays dits "en développement". Si, dans le passé, l'entreprise coloniale visait à exploiter les biens et matières premières, l'amour et l'affection ressentis aujourd'hui pour les enfants et les personnes âgées donnent de nouveaux paramètres à la circulation des personnes dans le monde.

Comment ces changements sont-ils vécus et interprétés par les employés et employeurs impliqués dans ce flux mondial qui remodèle l'opinion publique mondiale et les domaines les plus profonds de la vie?

\section{Le pays des "badanti"}

Barbara Da Roit et Carla Facchini (2010) montrent que, depuis la fin des années 1990, une nouvelle figure d'aide-soignante s'est imposée et a transformé radicalement l'organisation du travail de soins aux personnes âgées dépendantes en Italie. Badante est la "femme immigrée employée comme domestique qui a pour tâche de prêter assistance aux personnes âgées avec lesquelles elle vit, en général, et de les soigner." (p.11). ${ }^{6} \mathrm{Si}$ le nombre de badanti en Italie n'est pas connu, car la plupart sont en situation illégale, certaines estimations considèrent qu'elles sont entre 700 ooo et un million, un chiffre beaucoup plus important que celui des aides-soignantes de l'économie formelle.

Parler de badanti indique une rupture significative de l'image traditionnelle de la famille en tant qu'aidesoignante de personnes âgées. Da Roit et Facchini montrent qu'en Italie, contrairement à ce qui se passe dans d'autres pays d'Europe occidentale, le réseau familial étendu et le travail non-rémunéré des femmes ont été traditionnellement la source la plus importante de soins aux personnes âgées dépendantes. Si, comme le suggèrent Lewis (2001) et Knijn et Smit (2009), le soin informel familial est un aspect clé dans tous les modèles d'État-providence, ces chercheuses italiennes révèlent que ce type de soin est beaucoup plus présent en Italie, pays où le taux d'emploi des femmes est le plus faible d'Europe.7 Toutefois, la disponibilité de la famille pour ces soins est menacée non seulement par l'augmentation du niveau d'emploi des femmes et par la réduction du nombre d'enfants, qui caractérise aujourd'hui la structure familiale italienne, mais aussi par l'augmentation de l'espérance de vie, ce qui conduit à une augmentation du nombre de personnes âgées devenant ainsi plus dépendantes. Ces processus se sont produits dans plusieurs pays européens, mais, en Italie, le retard pris par les politiques publiques liées à la vieillesse est l'une des plus fortes raisons invoquées pour expliquer l'émergence et l'importance des badanti. Le développement

\footnotetext{
4 Le processus inverse existe également, qui vise à promouvoir le départ de personnes âgées des pays riches vers les pays pauvres, afin qu'ils puissent survivre avec une somme d'argent inférieure à celle de leur pension dans leur pays d'origine.

5 Ces stéréotypes ne sont pas seulement perpétués par les forces politiques et nationalistes de droite, mais aussi par des organisations conservatrices de gauche qui combinent la défense des mécanismes disciplinaires de l’État-nation (en particulier le système de protection sociale et de retraite centralisé) et les programmes anti-immigration.

6 "Badante" est donc le nom donné en italien à l’immigrée qui prête assistance aux personnes âgées et "badanti” est son pluriel.

7 À ce sujet, voir Scrinzi (2004); Sarti (2004); Russo (2008); Da Roit \& Facchini (2010); Castegnaro (2002); Ambrosini \& Cominelli (2005)
} 
de l'activité des soins à domicile, dans cette zone grise du travail illégal, représente la possibilité d'assurer l'accès à ce type de service à une plus grande partie de la population et de transformer l'Italie en un pays de badanti.

Le marché des soins aux personnes âgées en Italie a subi un changement. Les recherches montrent que depuis les années 1990 les immigrées venant d'Amérique latine (notamment de Bolivie, d'Équateur et du Pérou) et d'Asie (en particulier des Philippines et du Sri Lanka) ont été remplacées par des immigrées d'Europe de l'Est. Comme me l'a dit une Italienne ayant embauché des femmes immigrées pour s'occuper de ses parents âgés:

On veut plutôt les immigrantes d'Europe de l'Est que celles d'Amérique latine ou des Philippines parce qu'elles ont un niveau d'éducation beaucoup plus élevé, davantage d'expérience dans le traitement des personnes âgées et, surtout, elles ont déjà un certain âge. Elles n'ont pas besoin de s'occuper de leurs enfants, elles ne cherchent pas de petits amis et elles ne font pas la fête. Bref, elles se consacrent au travail.

Les raisons évoquées pour expliquer l'immigration d'Europe de l'Est sont liées à la désintégration du système socio-économique de ces pays, qui appartenaient autrefois à l'Union soviétique, et aux difficultés de la transition d'une économie planifiée à une économie de marché.

Quand Barbara Da Roit et Carla Facchini (2010) cherchent à retracer le profil des aides-soignantes, elles montrent que 70\% des interviewées proviennent d'Europe de l'Est, en particulier d'Ukraine, de Moldavie et de Roumanie, ${ }^{8}$ et qu'elles sont plus âgées que celles venant d'Amérique, d'Afrique et d'Asie: un tiers d'entre elles ont plus de 50 ans. Elles sont mariées et ont des enfants. Leur niveau d'éducation est plus élevé: près de la moitié des personnes interviewées ont une licence $(30,2 \%)$ ou un master $1(22,7 \%)$. Elles veulent rester une courte période en Italie et n'obtiennent pas facilement leurs papiers. Elles veulent gagner beaucoup d'argent en un laps de temps très court. Ces traits les différencient nettement des immigrées venant d'autres régions, qui sont jeunes, célibataires et ont un projet d'immigration à long terme, pour s'installer en Italie et y faire venir leur famille.

Parler de l'Europe de l'Est ne veut pas dire méconnaître la différence entre les immigrants des différents pays composant cette région. Castagnone (2007) souligne avec beaucoup de sensibilité les différences entre les aides-soignantes venues de Roumanie et d'Ukraine

Deux autres raisons sont souvent invoquées pour expliquer le taux élevé d'émigration de femmes d'Europe de l'Est: le chômage ou les bas salaires et le rôle qu'elles occupaient dans le cycle de la vie familiale: mariées ou veuves, avec des enfants adultes, et grands-mères, dans la plupart des cas.

Plusieurs études ont montré que les femmes immigrées ne proviennent pas de couches sociales basses, dans leur pays d'origine. ${ }^{9}$ Il leur faut un minimum de capital culturel pour réaliser leur projet d'immigration, notamment si elles ne possèdent pas un réseau de relations pouvant les accueillir à l'étranger. Il est tout de même frappant de constater le haut niveau d'éducation des aides-soignantes venant des différents pays d'Europe de l'Est.

Danya, ma principale interlocutrice, a 52 ans. Elle était professeure d'histoire en République de Moldavie et gagnait 40 euros par mois. À Bologne, en tant qu'aide-soignante d'un homme de 82 ans depuis 18 mois, elle touche un salaire mensuel de mille euros et peut en envoyer environ 800 par mois à sa famille. Comme elle me l'a dit, elle a peu de frais en Italie, car elle est logée et nourrie dans la maison où elle travaille. Pendant ses heures de repos hebdomadaire du dimanche, elle fait du lèche-vitrine et se promène

8 Enquête menée en Lombardie, comportant des entretiens avec 650 aides-soignantes membres du Syndicat Pensionati Italiano.

9 Voir, par exemple, Sassen (2003). 
avec ses amies. Elle est divorcée et envoie de l'argent en Moldavie pour financer les études de ses deux fils, qui sont déjà mariés, pères de famille et dont l'un aura un enfant le mois prochain. Elle économise encore de l'argent pour pouvoir passer deux semaines de vacances en Moldavie chez ce fils et connaître son petitenfant. Pendant cette période, il a été convenu que sa cousine viendra la remplacer en Italie.

Les études sur les badanti en Italie montrent que le temps libre de ces travailleuses est aussi limité que leurs chances de se développer et de participer à une vie sociale active. Elles constatent également que les badanti ont des relations intenses avec leur famille, surtout avec leurs enfants qui sont restés dans leur pays d'origine et, en général, elles concluent qu'il s'agit d'un travail solitaire effectué pour offrir une vie meilleure à leur famille.

L'amour des leurs et la réitération constante, de la part de toutes les femmes que j'ai interviewées, de ce que la décision de travailler en Italie est une façon de répondre aux besoins économiques de leurs enfants et petits-enfants sautent aux yeux, surtout quand ceux-ci sont adultes. Un peu comme si aider leur famille était la seule façon de justifier le projet de migration et la déqualification professionnelle qu'elles subissent en Italie.

Par ailleurs, l'étude menée par Francesco Vietti (2010) auprès d'aides-soignantes de Moldavie travaillant en Italie révèle des stéréotypes négatifs concernant la femme décidant d’émigrer. Les déplacements sont perçus comme le résultat d'un mélange à la fois d'égoïsme et de désir de s'enrichir, menant à l'abandon des obligations et des principes éthiques qui devraient régir les relations familiales. Cela explique peut-être pourquoi l'aide à la famille restée au pays est constamment invoquée.

Après plusieurs conversations, il est devenu clair qu'elles ont d'autres projets comme, par exemple, construire une maison dans leur pays ou aider leur père ou leur mari à ouvrir un petit commerce.

Les femmes péruviennes que j’ai interviewées sont plus jeunes et ont laissé de jeunes enfants au Pérou. Elles disent que ces derniers sont petits et que leur mère s'en occupe. De plus, elles entendent bien les faire venir en Italie le plus vite possible. Beaucoup disent qu'elles construisent ou veulent construire des maisons en Amérique pour y habiter pendant leur vieillesse ou pour les louer afin d'avoir un revenu pour leur retraite. Elles ajoutent qu'elles savent qu'après leur expérience en Italie, il leur sera très difficile de s'acclimater de nouveau au Pérou. Comme l'a dit l'une de mes interlocutrices: "Là-bas [au Pérou], il y a des hommes très machos et des femmes arriérées et rétrogrades." En quête de plus d'intégration, elles ne veulent plus vivre chez les personnes soignées et cherchent à être payées à l'heure pour faire des ménages ou s'occuper d'enfants ou de personnes âgées.

Le soin aux personnes âgées est composé de tâches variées, selon le degré d'autonomie fonctionnelle de la personne soignée. Seule l'une des femmes que j'ai interviewées s'occupait d'une personne âgée en situation de dépendance totale, car ses fonctions motrices et cognitives étaient fortement compromises. Les autres s'occupaient d'hommes et de femmes vivant seuls et ayant un certain degré d'autonomie fonctionnelle, ce qui leur permettait de se déplacer en ville, de se promener dans les parcs ou de rendre visite à des amis et à des membres de leur famille, car ils étaient accompagnés de l'aide-soignante.

Dans tous les cas, le travail de la badante implique de longues journées de travail, car les personnes âgées ne peuvent pas rester toutes seules et il faut une entente avec d'autres aides-soignantes ou avec les membres de la famille pour garantir les congés payés ou les heures de repos.

Les situations économique et professionnelle ainsi que le niveau éducationnel des personnes âgées varient, mais dans les cas que j'ai suivis, ce sont leurs filles qui embauchent et suivent le travail des badanti.

Les aides-soignantes que j'ai interviewées étaient très satisfaites de la relation établie avec les personnes âgées et leur famille, mais toutes avaient des histoires à raconter sur les enfants qui arrivent toujours en 
retard chez leurs parents le dimanche, faisant ainsi attendre les aides-soignantes qui ne peuvent pas laisser seules les personnes âgées. Elles ne savent donc jamais quand commence ou termine leur congé. Elles racontent les cas de filles qui apportent du linge sale chez leurs parents pour que la badante le lave et le repasse, ou des cas de réduction ou de retard du paiement convenu.

Lors de mes entretiens et conversations à ce sujet, j'avais toujours en tête que l'embauche d'une femme de ménage ou d'une badante est le privilège de personnes aisées. Or, la recherche de Monica Russo (2008) exige de reconsidérer cette hypothèse. Un extrait d'une interview qu'elle cite montre combien, en Italie, la badante est beaucoup plus populaire que l'on ne pourrait le croire:

Regardez, je suis ouvrière, nous économisons goo euros par mois... Je dépense presque tout pour payer le salaire de la badante, mais j'ai pas le choix. Ma mère ne peut rien faire toute seule. Franchement, si elle vit encore deux ou trois ans.... Qu'est-ce que je peux faire faire? Quitter mon travail pour m'occuper d'elle? Et puis, quand ma mère ne sera plus avec nous, est-ce que vous pensez que je pourrai trouver du travail à mon âge? J'ai pas le choix. Vivre avec le salaire de mon mari et payer la badante. C'est difficile, vous savez? Béni soit Dieu ! J'espère que la mère de mon mari ne va jamais tomber malade.

Le travail domestique en Italie semble avoir radicalement changé au cours de la dernière décennie. Comme le montre Francesca Scrinzi (2004), la quantité de foyers ayant une femme de ménage a largement augmenté grâce à la baisse des charges sociales. Au début des années 1980, avoir une femme de ménage fixe était un signe de distinction réservée aux familles de classes moyennes et supérieures. De nos jours, cela est répandu parmi les couches sociales à bas revenu.

Bien évidemment, une grande partie de la population italienne ne serait pas en mesure de payer ce genre de service si ce marché du travail était réglementé.

Le développement intense de ce marché est apparemment une stratégie gagnante pour les acteurs impliqués: les personnes âgées et leurs familles, les travailleurs immigrés et les policymakers. Pour les personnes âgées et leurs familles, il s'agit d'un avantage économique et affectif par rapport aux maisons de retraite ou d'autres types d'institutions de ce genre ; interner une personne âgée dans une maison de retraite est éthiquement intolérable et exige que les enfants prouvent qu' il n'y a aucune autre option possible. Pour le travailleur migrant, cela signifie la possibilité d'obtenir un salaire mensuel beaucoup plus élevé que dans son pays d'origine. La cohabitation permet non seulement d'économiser de l'argent, mais encore de se protéger contre les effets de l'illégalité. En ce qui concerne les politiques publiques, cela représente une réduction considérable des demandes auprès des services sociaux ciblant les personnes âgées dépendantes.

La littérature internationale sur le genre et l'immigration et la presse ont à maintes reprises dénoncé des abus: violence physique et sexuelle, journées de travail interminables, humiliations et restrictions de sortie de la maison de l'employeur. Comme le montre Parreñas (2001), les aides-soignantes, même celles qui ont leurs papiers, n'ont pas de droits. La presse dénonce régulièrement des cas de retrait des papiers des employées et de menaces de prison ou de reconduite aux frontières. L’idée du "retour de la servitude domestique", de l"'esclavage moderne" ou de l'homo sacer semble se concrétiser.

Toutefois, parler avec les badanti nous pousse à reconsidérer ces situations: elles racontent avec joie leurs achats de cadeaux et d'électroménagers quand elles se préparent à rentrer dans leur pays d'origine. Heureuses, elles échangent des recettes et commentent combien il est facile d'accomplir les tâches ménagères en Italie. Elles appellent la personne âgée papi ou mamie et se souviennent sans regret des difficultés vécues avant de se décider à aller travailler en Italie.

Monica Russo (2008) a montré avec beaucoup de sensibilité que les situations vécues par les badanti sont beaucoup plus ambigües qu'on ne l'imagine. Elle attire l'attention sur le processus de négociation entre les parties: la badante avec la personne âgée, mais aussi avec la famille de cette dernière, qui l'emploie. 
Dans ces relations, différemment de celles réglementées par des contrats de travail formels, des facteurs psychologiques et émotionnels sont toujours présents. Les parties doivent avoir une interactivité dynamique et contextuelle parce que c'est dans l'exercice des tâches quotidiennes que les droits et les devoirs des différents acteurs sont définis.

Comme le montrent Da Roit et Facchini (2010), ce nouveau modèle de soins, basé sur le travail de femmes immigrées, présente des tensions et des contradictions. La gestion des soins est négociée de manière privée entre la famille de la personne âgée et les immigrées. Cette négociation exige de la confiance de part et d'autre, un partage d'intérêts et des arrangements délicats impliquant de l'argent et du temps investi dans le travail. Souvent, la relation de travail est contestée par l'une ou l'autre partie du fait du manque ou de l'abus de confiance et, par conséquent, il en résulte une situation d'instabilité et d'insécurité pour les deux parties.

Bref, la disponibilité limitée des services sociaux et de main-d'œuvre à bas coût et une mauvaise réglementation des flux migratoires créent un marché d'aides-soignantes immigrées. Cette transformation comporte une transition d'un modèle de soins familiaux vers un modèle basé sur le travail d'immigrés au sein de la famille où les enfants de la personne âgée cherchent des aides-soignantes. Le travail de soin organisé comme travail domestique informel est une réponse à la nécessité économique de soins. Le salaire moyen sur le marché informel tourne entre 800 et 1 ooo euros par mois pour un service exercé pratiquement 24 heures sur 24 pendant au moins six jours par semaine. Ironiquement, Ambrosini et Cominelli (2005) surnomment le rapport entre immigration et État-providence Welfareleggero (état-providence allégé), dans ces cas.

\section{L'ethnicité des rapports sociaux de genre}

Le sexe, la race et la culture sont des composantes essentielles pour comprendre le processus de déplacement des frontières qui donne une nouvelle configuration aux soins des personnes âgées de ce que l'on appelle les pays du Nord.

À partir d'une étude sur les agences de travail et de formation des femmes de ménage à Gênes, Scrinzi (2004) décrit les modes d'interaction entre ces agences, les immigrées cherchant un travail domestique et leurs employeurs potentiels. Les employées des agences inscrivent sur des fiches le type de service requis (babysitting, tâches ménagères, nettoyage) et le salaire proposé. Ces fiches répertorient aussi les caractéristiques physiques et de personnalité des personnes recherchées: la nationalité, l'âge, la couleur de peau et d'autres caractéristiques comme: "une personne de confiance", "honnête", "sérieuse", "très patiente", "douce", "polie", "fiable", de préférence "italienne" plutôt "que d’Amérique du Sud”, "jeune fille de l'Est", "pas de femme de couleur", ou alors "une femme de couleur", des "jeunes d'environ 25 ans", femmes de "plus de 30 ans, entre trente et quarante ans", "pas de femme jeune", "une femme mûre".

D’après l'auteur, les critères énoncés en détail par les employeurs pour décrire le travailleur domestique ou l'aide-soignant montrent à quel point cette relation d'emploi est considérée comme une relation personnelle, dans laquelle une des deux personnes impliquées se sent habilitée à choisir, à juger et à veiller sur l'autre. Lors des conversations téléphoniques, ils utilisent des mots décrivant les travailleuses, qui sont souvent au chômage et attendent un appel proposant du travail, comme des marchandises. Ils montrent le corps ou des parties du corps de ces femmes et l'immigrée en attente d'emploi sont décrites comme "bosseuse", "une personne en bonne santé", "costaude, un caractère fort et honnête". Dans cette rhétorique, typique du discours raciste, les individus sont rabaissés à la fonction qui leur a été imputée. Leurs qualités personnelles, morales, intellectuelles et sociales sont réduites à une identité physique ou biologique. Dans d'autres cas, certaines qualités sont renforcées "féminine", "aimable", "gentille", "très patiente", "c'est une belle fille, propre, elle a bonne mine", "elle a un bon caractère". 
À partir de ces interactions, les agences montrent une certaine ambivalence, à l'instar de leur positionnement sur l'interface entre l'offre et la demande de travail. Elles aident à alimenter et légitimer la discrimination, à transcrire les désirs des employeurs et à les prendre comme critères pour naviguer à l'intersection entre offre et demande. La compétence pour les soins n'est pas simplement un fait de la féminité, mais un rôle culturel naturalisé chez les femmes immigrées, ce qui se manifeste dans les conversations transcrites: "parfait pour le nettoyage, comme tous les Marocains"; "les Sénégalais sont vraiment super avec les enfants", "les Sud-Américains ont beaucoup d'humanité, ils savent comment communiquer avec les personnes âgées".

La culture est perçue comme une identité statique et essentielle, extraite de l'historicité des relations sociales. Scrinzi suggère également que la naturalisation de la différence est constitutive de l’idée de féminité "respectable". Il convient de rappeler que la féminité de la femme blanche occidentale a historiquement été constituée non seulement en opposition à l'idée de virilité, mais aussi en opposition à la sexualité et à la féminité de la femme "noire" des colonies. De même, conclut-elle, les conceptions sur les aides-soignantes immigrées créent une frontière entre les "Européennes" et celles auxquelles cette double condition, de femme et d'Européenne, est refusée. Ce sont ces limites et l'établissement de nouvelles règles sur "être femme" en opposition aux immigrées des pays du Sud qui permettent aux femmes européennes d'occuper des positions de pouvoir. ${ }^{10}$

D’autres stéréotypes (comme le résultat de l'imbrication des différences nationales et culturelles concernant le sexe) délimitent les catégories accusatrices caractérisant les conflits sur le marché du travail entre les immigrés eux-mêmes. Parmi la hiérarchie des éléments caractérisant une bonne aidesoignante se trouvent le niveau d'éducation et le fait d'être originaire d'Europe de l'Est. Pourtant, les jeunes filles d'Amérique latine que j'ai interviewées me disent que les femmes d'Europe de l'Est sont froides, calculatrices, prêtes à travailler sans salaire parce qu'elles souhaitent épouser la personne âgée pour obtenir leurs papiers et mener une vie tranquille. De même, elles ne comprennent jamais la vie des femmes asiatiques qui, selon elles, seraient toujours souriantes sans raison claire, et pensent que les Africaines viennent d'une société où il n'y a ni propreté, ni éducation. Comme me l'a dit une Péruvienne: "Nous savons ce qu'est l'amour et le respect du traitement des personnes âgées: nous apprenons comment prendre soin des personnes âgées et nous les considérons comme un membre de notre famille". De cette façon, elles cherchent à redéfinir et à créer d'autres conventions relatives à l'ethnicité, à la nationalité et au genre.

\section{Considérations Finales}

Du point de vue structurel, le flux de travailleurs de pays pauvres vers des pays plus riches pour exercer des métiers de soins aux personnes âgées est un effet de la croissante division des richesses et du travail dans un monde mondialisé. C'est ce mouvement qui rend l'immigration illégale susceptible de prison et de déportation. Il établit les relations de travail recréant des pratiques esclavagistes au cœur des riches démocraties des pays du Nord. En ce sens, les immigrées aides-soignantes de personnes âgées sont une expression de l'homo sacer, en accord avec la façon dont Brett Neilson reprend les positions d'Agamben et de Foucault pour établir une relation entre vieillesse, biopolitique et mondialisation.

\footnotetext{
10 D’après Scrinzi, il en va de même sur le marché transnational du sexe. La description de prostituées des pays de l'Est les transforme en victimes passives de proxénètes internationaux. Cela vise à prouver l'appauvrissement des femmes de l'Est tout en créant une frontière entre les “femmes européennes" et celles qui sont refoulées. Sur ce thème, voir Piscitelli (2007) et le dossier “Gênero no Tráfico de Pessoas” (Le Genre dans la traite de personnes”) paru aux “Cadernos do Pagu”, n. 31, 2008.
} 
L'ethnographie réalisée propose des éléments pour la complexification de ces analyses portant une marque structurelle. Nous avons voulu montrer que les immigrées illégales, employées comme aidessoignantes de personnes âgées, redéfinissent les formes prises par la dépendance et donnent un contenu et des sens nouveaux aux relations familiales, aux droits et obligations de l'État et à la vie urbaine. Les aides-soignantes offrent non seulement de nouvelles significations à la présence de personnes âgées dans les espaces publics, mais elles transforment aussi les domaines privés en une sorte de "microcosme exotique en Europe", expression suggérée par Scrinzi (2004) pour caractériser ce nouveau contexte.

Les débats sur l'immigration et le travail domestique imposent à la politique et à la théorie féministe une réouverture de la discussion sur la division sexuelle du travail au moment où les faiblesses de l'État-providence incitent les femmes des pays du Nord à explorer les nouvelles frontières du travail domestique.

Selon Barbara Ehrenreich et Arlie Hochschild (2003), ce flux migratoire signifie une révolution des sexes dans le monde entier, quoiqu'une révolution contradictoire et ambivalente. Basée sur une redistribution du travail reproductif, elle modifie les rapports de pouvoir, mais pas la division du travail homme-femme, car l'homme n'accomplit pas toutes les tâches ménagères, ni tous les soins. En tant qu'aides-soignantes, les femmes immigrées arrivent à avoir un certain poids économique et de décision économique au sein de leur famille: en effectuant les tâches ménagères, elles participent au marché du travail. Cependant, les formes d'exploitation ont atteint une dimension terrifiante et les familles, bien comme les employées immigrées illégales qu' elles emploient, se retrouvent dans une situation d'illégalité.

Or, sans nier le caractère dramatique de l'immigration illégale, parler de pratiques esclavagistes est doublement compliqué. D’une part, cela dissout la complexité des situations concernées et, de l'autre, cela destitue les aides-soignantes de leur capacité de négociation, comme c’est le cas pour le marché transnational de la prostitution. Comme le montre Scrinzi (2004), la description de prostituées venues de pays pauvres uniquement comme des victimes passives de trafiquants de même nationalité a pour fonction d'exalter la centralité du modèle européen de renforcement du pouvoir de la femme, créant ainsi une frontière entre les "femmes européennes" et celles auxquelles on refuse cette condition. Cela masque le fait que pour beaucoup de femmes, les réseaux de prostitution sont les seules structures disponibles pour migrer vers l'Union européenne. L'auteur suggère que faire la morale du système de prostitution en ne voyant en lui que ses pratiques violentes, signifie oublier que ces personnes sont les pôles "méprisables" contenant l'idée de féminité "respectable" et gravitant autour d'elle.

De même, l'on peut dire que limiter l'attention portée au phénomène des immigrées aidessoignantes de personnes âgées aux seuls cas sensationnels "d'esclavage moderne" revient à ignorer que l'aide-soignant domestique est un élément actif dans la production de la "famille respectable", celle qui permet aux personnes âgées de rester chez elles en recrutant, payant ou organisant le travail d'aidessoignantes de personnes âgées, immigrées, pauvres et privées de leurs droits. Ces dernières, qu'elles vivent dans l'illégalité ou non, sont des pôles méprisables car elles ont abandonné leur famille, leurs jeunes enfants ou leurs parents âgés en quête de meilleures conditions de vie dans d'autres pays.

C'est aussi cacher le fait que l'entrée sur le marché du travail des soins de personnes âgées est l'une des seules formes d'immigration dans l'Union européenne pour les femmes plus âgées à la recherche de meilleures conditions de vie que celles qu'elles connaissent dans leur pays. 
L'Italie a connu des changements dans ses espaces publics où l'on remarque de plus en plus la présence de ces deux populations indésirables, personnes âgées et immigrés, présence qui, comme nous l'avons montré, redéfinit les espaces publics, la vie domestique et les relations entre générations dans la famille. Est-ce un changement transitoire? L’immigration sera-t-elle freinée? Y aura-t-il un changement dans la législation sur les aides-soignantes? Il est très difficile de le savoir. Tout au moins, nous sommes sûrs que les recherches que nous menons et les textes que nous produisons, où nous proposons des réponses aux difficultés identifiées, joueront un rôle central dans le destin des personnes âgées et de leurs aides-soignantes.

Traduction: Bertrand Borgo

Reçue, 30/03/2015, approuvée, 01/06/2015

\section{Bibliographie}

AGAMBEN, Giorgio. 2002. Homo sacer - o poder soberano e a vida nua. Belo Horizonte: Editora UFMG.

AMBROSINI, Maurizio; COMINELLI, Claudia. (orgs). 2005. Un'assistenza senza confini. Welfare 'leggero', famiglie in affanno, aiutanti domiciliari immigrate. Rapporto 2004. Milano: Fondazione Ismu. Regione Lombardia. Osservatorio Regionale per l'integrazione e la multietnicità.

CASTAGNONE, Eleonora et al. 2007. "Madri migranti - Le migrazioni di cura dalla Romania e dall’Ucraina in Italia: percorsi e impatto sui paesi di origine”. Roma: CeSPI - Centro Studi di Politica Internazionale FIERI - Forum Internazionale ed Europeu di Ricerche sul’ Immigrazione. Working Papers 34. Mimeo.

CASTEGNARO, A. 2002. "La rivoluzione occulta nell'assistenza agli anziani: le aiutanti domiciliari". Studi Zancan-Politiche e Servizi Alle Persone, 2: 11-34.

DA ROIT, Barbara; FACCHINI, Carla. 2010. Anziani e Badanti - Le differenti condizioni de chi è accudito e di chi accudisce. Milano: Franco Angeli.

DEBERT, Guita Grin. 1999. A reinvenção da velhice: socialização e processos de reprivatização do envelhecimento. São Paulo: EDUSP Editora da Universidade de São Paulo.

EHRENREICH, Barbara; HOCHSCHILD, Arlie Russel. 2003. "Introduction”. In: _-_- (orgs.), Global women nannies, maids, and sex workers in the new economy. New York: Henry Holt and Company. pp.1-13

FOUCAULT, Michel. 1976. Histoire de la sexualité, t. I, La volonté de savoir. Paris: Gallimard.

HOCHSCHILD, Arlie Russel. 2003. "Love and gold”. In: Barbara Ehrenreich; Arlie Russel Hochschild (orgs.)., Global women - nannies, maids, and sex workers in the new economy. New York: Henry Holt and Company. pp.15-30.

KNIJN, Trudie; SMIT, Arnoud. 2009. "Investing, facilitating, or individualizing the reconciliation of work and family life: three paradigms and ambivalent policies". Social Politics, 16(4): 484-518.

LEWIS, Jane. 2001. "The dDecline of the male breadwinner model: implications for work and care". Social Politics, 8(2): 152-169.

NEILSON, Brett. 2003. "Globalization and the biopolitics of aging". The New Continental Review, 3(2): 161-186.

PARREÑAS, Rhacel. 2001. "Transgressing the national state: partial citizenship and 'imagined (global) community' of migrant Filipina domestic workers". Signs: Journal of Women in Culture and Society, 26(4): 1119-1154. 
PISCITELLI, Adriana. 2007. "Corporalidades em confronto: brasileiras na indústria do sexo na Espanha". Revista Brasileira de Ciências Sociais, 22(64): 17-32.

RABINOW, Paul; ROSE, Nikolas. 2006. "Thoughts on the concept of biopower today”, BioSocieties, 1(2): 195217.

RUSSO, Monica. 2008. Donne migranti a Modena: il lavoro di "badante" tra vincolo e risorsa. Thèse de Doctorat, Cooperazione Internazionale e Politiche per lo Sviluppo Sostenibile. Ciclo XIX. Alma Mater Studiorum. Università di Bologna, Bologna.

SARTI, Raffaella. 2004. "Noi abbiamo visto tante città, abbiamo un'altra cultura'. Servizio domestico, migrazioni e identità di genere in Italia: uno sguardo di lungo periodo". Polis, 1: 17-46.

SCRINZI, Francesca. 2004. "Professioniste della tradizione. Le donne migranti nel mercato del lavoro domestico". Polis, 1: 107-136.

SPINELLI, Elena. 2003. "Badanti: donne come noi". La Rivista di Servizio Sociale, 2: 39- 66.

SASSEN, Saskia. 2001. "Entrapments rich countries cannot escape: governance hotspots". Disponible sur: http://www.theglobalsite.ac.uk/times/109Sassen.ttm . 2003. "Géo-économie des flux migratoires". Esprit, 300: 102-113.

VIETTI, Francesco. 2010. Il paese delle badanti. Roma: Meltemi Editore.

\section{Guita Grin Debert}

Unicamp, Institut de Philosophie et Sciences Sociales, Département d'Anthropologie, Campinas/SP, Brazil

ggdebert@uol.com.br 